\title{
Neurochemical mechanisms of stimulation-produced analgesia: Comparison of tests involving tonic and phasic pain
}

\author{
BEVERLY E. THORN and KENNETH E. PLOTKIN \\ The Ohio State University, Columbus, Ohio
}

\begin{abstract}
Male albino rats were implanted with bipolar electrodes into the periaqueductal gray. Focal brain stimulation-produced analgesia (SPA), as measured by the formalin test and the tail-flick test, was challenged with either naloxone ( $3 \mathrm{mg} / \mathrm{kg}$ ) or methysergide $(1 \mathrm{mg} / \mathrm{kg})$. Naloxone failed to reverse SPA when the formalin test was used; mean pain ratings remained close to analgesic values after the opiate antagonist was administered. However, methysergide (a serotonin receptor blocker) did attenuate the analgesia significantly. When the tail-flick test was used to measure antinociception, both methysergide and naloxone attenuated the analgesia. The data suggest that a nonopioid (serotonergic) system is involved in the reduction of both tonic and phasic pain, whereas a naloxone-reversible opiatergic system is also involved in phasic pain.
\end{abstract}

Nociceptive challenges used in the laboratory usually involve very brief pain without tissue damage, sometimes referred to as phasic pain (Dennis \& Melzack, 1979). Such tests involve noxious thermal, electrical, or mechanical stimuli (e.g., the tail-flick test) (D'Amour \& Smith, 1941).

Tonic pain stimuli are associated with continuous pain from tissue damage (Dennis \& Melzack, 1979). This type of pain is seldom induced in the laboratory. However, there are laboratory procedures being used which do inflict tissue damage, and hence involve more tonic pain stimuli. Dubuisson and Dennis (1977) have devised an animal model for tonic pain, called the formalin test. In this test, temporary tissue damage is inflicted on a rat's forepaw by the injection of dilute formalin.

Opiate analgesic drugs are potent pain relievers, having their mechanism of action at the opiate receptor in the brain. Electrical stimulation of the midbrain central gray in rats produces antinociception similar to morphine analgesia (Reynolds, 1969). Although the neural mechanism for stimulation-produced analgesia (SPA) is probably not solely opiatergic, there is considerable evidence for the involvement of the endogenous opiate system in the substrate of SPA (Bennett \& Mayer, 1979; Hughes, 1975; Mayer \& Hayes, 1975).

There is some evidence to suggest that different types of pain may be mediated by different pain suppressing systems. The opiate antagonist, naloxone, has different actions, depending upon the type of pain stimulus: Morphine analgesia has been shown to be reversed by nalox-

The authors wish to thank the Comparative and Physiological Area in the Department of Psychology at Ohio State University for their kind assistance. The authors' mailing address is: Department of Psychology, Ohio State University, Townshend Hall, 1885 Neil Avenue Mall, Columbus, OH 43210-1222. one when phasic challenges such as the tail-flick or the hot plate tests are used (Akil, Mayer, \& Liebeskind, 1976), but naloxone is ineffective in reversing morphine analgesia as measured by the formalin test (Dubuisson \& Dennis, 1977). Additionally, naloxone has been reported to reverse or attenuate focal brain stimulation-produced analgesia as measured by phasic challenges such as the tail-flick test (Akil et al., 1976; Swajkoski, Mayer, \& Johnson, 1981), whereas naloxone does not increase the stimulation threshold necessary for analgesia when the formalin test is used (Dennis, Choiniere, \& Melzack, 1980). These findings suggest that although attenuation of phasic pain involves a naloxone-reversible opiate mechanism, suppression of tonic pain may rely on other neural substrates.

Serotonin (5-hydroxytryptamine, 5-HT) has also been shown to be differentially involved in tonic and phasic pain. Serotonin antagonists produce variable results, depending upon the type of nociceptive challenge involved. Using phasic tests of nociception, such as the tail-flick test, serotonin antagonists attenuate morphine-induced analgesia (Akil \& Mayer, 1972; Dennis et al., 1980; Yaksh, 1979). With the formalin test (tonic pain), serotonin antagonists have a more variable effect: although serotonin antagonists attenuate analgesia produced by $10 \mathrm{mg} / \mathrm{kg}$ morphine, they potentiate the effect of a subanalgesic does of morphine $(2.5 \mathrm{mg} / \mathrm{kg})$, rendering an analgesic action (Dennis \& Melzack, 1980).

The investigations discussed above point to differences in tonic and phasic pain and their suppression. Given the information already known about differential naloxone reversal of stimulation-produced analgesia (SPA) with tonic and phasic pain tests, it would be of interest to similarly test the effect of 5-HT antagonists on SPA using both tonic and phasic pain tests. 
This experiment was designed to investigate the effect of both a morphine antagonist (naloxone) and a 5-HT antagonist (methysergide) on stimulation-produced analgesia using both tonic and phasic pain tests. It was hypothesized that naloxone would reverse SPA when the tail-flick test was used but not when the formalin test was used (as shown previously by Akil et al., 1976, and Dennis et al., 1980). It was also hypothesized that methysergide would reverse SPA when the tail-flick test was used (based on inferences made from results of studies using morphineinduced analgesia) (Akil \& Mayer, 1972; Dennis et al., 1980; Yaksh, 1979), but not when the formalin test was used (based on the anecdotal observations of Dennis et al., 1980 , that a serotonin antagonist did not effect SPA as measured by the formalin test).

\section{METHOD}

\section{Subjects}

The subjects were 24 experimentally naive male albino Holtzman rats, weighing $350-450 \mathrm{~g}$ at the time of surgery. The animals were housed in individual cages and had ad-lib access to Purina Rat Chow and water. They were kept on a 12-h light cycle, with lights on from 8 a.m. to 8 p.m.

\section{Materials and Apparatus}

Drugs. The formalin used for behavioral testing was a $5 \%$ sollution (diluted with distilled water), and each rat received a 0.05 $\mathrm{ml}$ subcutaneous injection in the dorsal surface of a forepaw prior to observation. The two chemical antagonists, naloxone and methysergide, were dissolved in $9 \%$ saline solution and administered at a volume of $1 \mathrm{ml} / \mathrm{kg}$ body weight. Both substances were administered intraperitoneally, naloxone at a dose of $3 \mathrm{mg} / \mathrm{kg}$ and methysergide at $1 \mathrm{mg} / \mathrm{kg}$. The doses of the chemical antagonists were chosen to maximize the possibility of antagonistic effects while at the same time avoiding toxicity (especially relevant to methysergide). The dose of naloxone has not been shown to be critical for reversal of SPA as long as it is within .1-10 mg/ $\mathrm{kg}$ (Cannon, Prieto, Lee, \& Liebeskind, 1982).

Apparatus. Focal brain stimulation was delivered by a Grass S6C stimulator and consisted of trains of biphasic, rectangular-wave pulse pairs, with $100-\mu \mathrm{sec}$ pauses between pulses. Stimulation frequency was $50 \mathrm{~Hz}$, and intensity was between 10 and $20 \mu \mathrm{A}$, as necessary to induce analgesia.

For the tail-flick test, the rat was placed in a cylindrical chamber with openings for the head, tail, and electrode leads. Noxious radiant heat stimulation was delivered via a standard tail-flick device, constructed in this laboratory and described in detail in previous work (Thorn-Gray, Johnson, \& Ashbrook, 1982; Williams \& Thorn, 1984).

The observation chamber for the formalin test was a $75 \times 36$ $\times 30 \mathrm{~cm}$ glass aquarium. The rats were allowed free movement while attached to the stimulator leads.

\section{Procedure}

Surgery. The rats were anesthetized with sodium pentobarbital (Nembutal), supplemented by atropine sulfate, and one bipolar stimulating electrode was stereotaxically implanted into the mesencephalic gray matter of the brain. Electrodes were bipolar, made of twisted stainless steel wire coated with insulation except at the tip (Plastic Products Co., MS303/1; electrode diameter = $0.25 \mathrm{~mm}$ ). Standard stereotaxic techniques were used: target coordinates were $6.6 \mathrm{~mm}$ caudal to bregma, $1.8 \mathrm{~mm}$ lateral to midline, and $5.5 \mathrm{~mm}$ ventral to top of the skull (Pellegrino \& Cushman.
1967). Electrodes were angled at $17^{\circ}$ from the vertical plane of the midline. At least 1 week was allowed for recovery from surgery before testing.

Experimental groups. All animals were tested with both nociceptive challenges (tail-flick test and formalin test), with the order of presentation counterbalanced. A recovery period of 1 week was allowed between tests. The rats were grouped randomly according to which antagonist they were administered: naloxone, Group 1 (N =9); methysergide, Group $2(\mathrm{~N}=9)$; saline, Group $3(\mathrm{~N}=6)$.

Criteria for analgesia. Because analgesia was a necessary condition in order to test the effects of antagonists, criteria for analgesia were arbitrarily set so that each animal could be evaluated prior to injection of an antagonist. With the tail-flick test, antinociception increases the latency to remove the tail from the heat source. In the tail-flick test, $30 \%$ above baseline tail-flick latency was determined to represent analgesia. With the formalin test, baseline pain index is represented by a high numerical rating and antinociceptive responses result in decreased scores of pain responsivity. In the formalin test, a pain rating below $50 \%$ of baseline values was determined to represent analgesia.

Formalin test. The formalin test procedure was adapted from Dubuisson and Dennis (1977). After a subcutaneous injection of formalin into the dorsal surface of a forepaw, a wire lead was attached to the rat's electrode assembly and the rat was placed in the observation chamber for $1 \mathrm{~h}$. Pain responses were recorded according to a predetermined set of rating criteria based on the position of the afflicted paw relative to the floor (Dubuisson \& Dennis, 1977):0-full weight is placed on both forepaws; 1 - the injected paw is touching the floor but is not dorsoflexed, and is being favored; 2 -the injected paw is lifted off of the floor; and 3-the rat licks or bites the injected paw. Grooming and rearing behaviors were given a rating of 1 .

Pain responses were recorded every $15 \mathrm{sec}$ and averaged to give a pain index for each observation period. Following a 5-min baseline observation period, PAG stimulation was administered in a 45$\mathrm{sec}$ train of impulses. Thereafter, pain ratings were recorded for $5 \mathrm{~min}$ (a poststimulation observation period), in order to assess the degree of antinociception. After observation for SPA, the rat was injected intraperitoneally with the appropriate antagonist (from coded bottles to ensure experimenter blind conditions). Ten minutes were allowed for drug onset latency (as deemed necessary by pilot work in this laboratory). Electrical brain stimulation followed, and pain responses were observed for $5 \mathrm{~min}$ (a postantagonist observation period).

Tail-flick test. The tail-flick test was modified from the procedure of D'Amour and Smith (1941). Prior to testing, the rats were habituated to the cylindrical restraints for two periods of $1 \mathrm{~h}$ each. On the testing day, the stimulator lead was attached and baseline latency was determined. Intensity of the light bulb was adjusted to give a tail-flick latency of 3.5 to $4.5 \mathrm{sec}$ without brain stimulation. Four consecutive trials within baseline latency range constituted baseline.

A 45-sec train of impulses to the brain was administered, with a trial commencing $30 \mathrm{sec}$ after the onset of stimulation. The average tail-flick latencies 2 min after stimulation were used to measure nociception. This procedure was repeated four times, with 2 min between trials. The rat was then injected intraperitoneally with the appropriate antagonist, and $10 \mathrm{~min}$ were allowed for drug onset latency before another testing period was begun. Average tailflick latencies for this postantagonist observation period were then derived.

Histology. At the conclusion of testing, the rats were given lethal doses of sodium pentobarbital and intracardially perfused with $0.9 \%$ saline followed by $10 \%$ formalin solution. Serial frozen sections $(40 \mu)$ were made of the brain regions where the electrode tracts were located. The sections were mounted on glass microscope slides and stained with thionin. A projection microscope was used to verify electrode placement. 


\section{RESULTS}

\section{Formalin Test}

Due to the subjective nature of pain ratings in the formalin test, random interrater reliability checks were conducted for 8 of the 24 subjects. A concordance of $89 \%$ was calculated from the average pain ratings for each period.

A $3 \times 3$ (group $\times$ condition) analysis of variance (ANOVA) with repeated measures was performed on the formalin test pain ratings and revealed a significant main effect for condition $[\mathrm{F}(2,21)=50.84, \mathrm{p}<.001]$. Planned comparisons revealed that the analgesia was not reversed by naloxone administration: There were no significant differences between pain responses after the administration of naloxone and the responses observed after brain stimulation. Methysergide attenuated, but did not completely reverse, the antinociceptive response to brain stimulation: Comparisons between pain responses after the administration of methysergide and responses after brain stimulation revealed significant differences $[\mathrm{F}(1,63)=8.65, \mathrm{p}<.01]$. However, postmethysergide values remained significantly different from baseline values $[F(1,63)=10.40, p<.01]$, indicating a decrease in the level of analgesia, which still proved more antinociceptive than baseline pain levels. Saline injection did not attenuate the analgesia produced by brain stimulation (see Figure 1).

\section{Tail-Flick Test}

A $3 \times 3$ (group $\times$ condition) ANOVA with repeated measures was performed on the tail-flick latencies and revealed a significant main effect for condition $[F(2,21)=$ $33.82, \mathrm{p}<.01]$. Planned comparisons revealed that analgesia, as measured by the TF test, was attenuated, although not completely reversed, by naloxone administration: TF latencies after naloxone administration were significantly different from latencies seen after brain stimulation $[\mathrm{F}(1,63)=6.62, \mathrm{p}<.05]$, but postnaloxone latencies also remained significantly different from baseline latencies $[F(1,63)=9.57, p<.01]$. Likewise, methysergide attenuated, but did not completely reverse, the analgesia produced by brain stimulation: After methysergide administration, there was a significant decrease in TF latencies from those observed after brain stimulation $[F(1,63)=12.94, p<.001]$, but postmethysergide values remained significantly different from baseline values $[F(1,63)=19.85, \mathrm{p}<.001]$. Brainstimulation-produced analgesia was not attenuated by the injection of saline (see Figure 1)

\section{Histology}

Microscopic evaluation of electrode tracts verified 22 of 24 placements within the PAG. The two remaining histologies were unidentifiable due to poor perfusions. Of the 22 PAG placements, 14 were located within the dorsal or lateral aspect of the PAG (as far ventral as the level of the dorsal raphe nucleus), and 8 were located within

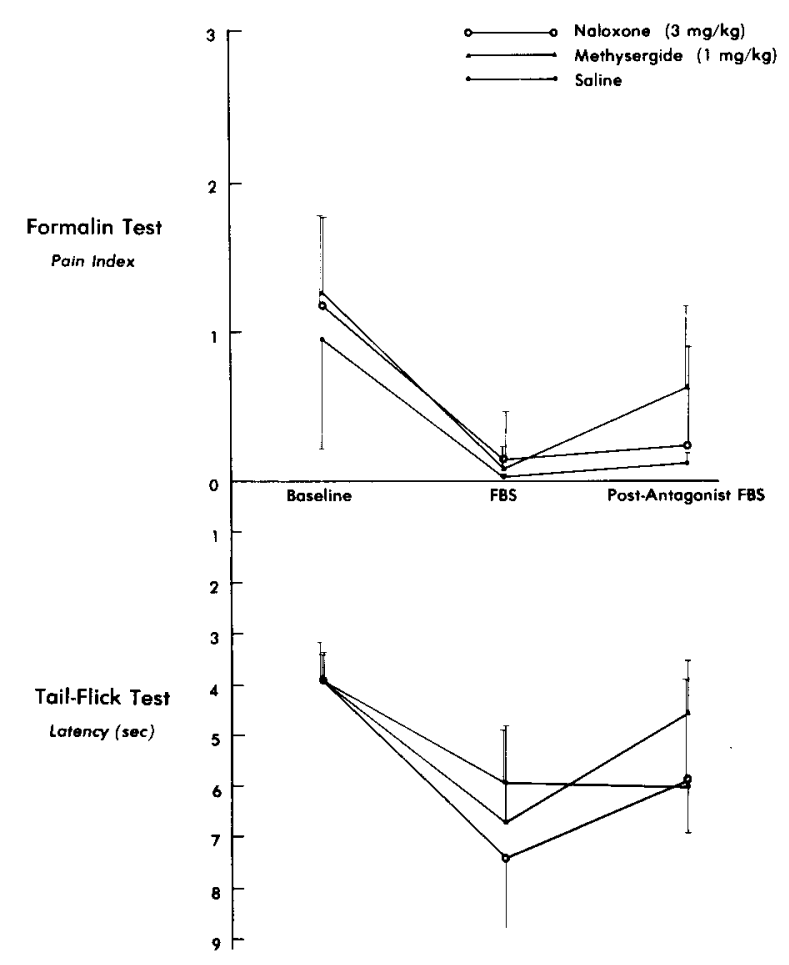

Figure 1. Average pain indices in the formalin test (upper graph) and latencies in the tail-flick test (lower graph). Open circles represent animals receiving naloxone as the potential chemical antagonist, and triangles represent animals receiving methysergide as the potential chemical antagonist. Closed circles represent the salineinjected control groups for each study. Pain indices and tail-flick latencies are shown for baseline, postfocal brain stimulation (FBS), and postantagonist FBS periods. Vertical bars represent standard deviations.

the ventral portion (within or subadjacent to the dorsal raphe nucleus).

\section{DISCUSSION}

The data suggest that a nonopioid neurotransmitter is involved in the analgesia produced by stimulation of the PAG. The ability of methysergide to reverse or attenuate SPA in both the tail-flick and the formalin tests implies an important role for serotonin in the suppression of tonic and phasic pain.

The data also suggest that naloxone-reversible opiates are involved in the suppression of phasic, but not tonic, pain. as evidenced by naloxone's attenuation of analgesia when the TF test is used but not when the formalin test is used. These results are consistent with previous investigations of naloxone's differential effect on SPA when different nociceptive challenges are used (Akil et al., 1976; Dennis et al., 1980).

Previous reports of naloxone reversal or attenuation of SPA have been equivocal, some laboratories reporting that naloxone does not attenuate SPA (Yaksh, Yeung, \& Rudy, 1976), others showing that placement of the electrode may be an important factor in naloxone reversal (Prieto, Can- 
non, \& Liebeskind, 1983). These researchers have reported that when the stimulating electrode is located in the dorsal aspect of the PAG, naloxone reversiblity is not obtainable. However, electrodes located within or subadjacent to the dorsal raphe nucleus are much more responsive to naloxone. Although it would have been interesting to compare possible differential effects of dorsal versus ventral placements in the present investigation, electrode placement was not specifically manipulated. Only a small number of our placements ended in the extreme ventral location investigated by Prieto et al (1983). Visual inspection of the data did not reveal any systematic differences in antagonist effect between dorsal and ventral placements. It may be that more ventral placements would have resulted in a greater naloxone effect. Nevertheless, since all SPA sites were tested with both nociceptive challenges, the differential effects of naloxone on the tail-flick versus the formalin test is still a crucial finding.

Methysergide attenuation of SPA on both the formalin and tail-flick tests were new findings, not based on previous systematic research. Although Dennis et al. (1980) had observed that p-chlorophenylalanine did not reverse SPA when the formalin test was used, these results were anecdotal, aimed at a possible explanation of the results discussed in the investigation. Although it has been reported that 5-HT antagonists attenuate morphineinduced analgesia when phasic challenges are used (Akil \& Mayer, 1972; Dennis et al., 1980; Yaksh, 1979), it remains unclear whether brain-stimulation-produced analgesia is similar enough to morphine-induced analgesia to warrant such comparisons. It appears that the more research generated from investigations of brainstimulation-produced analgesia, the more indications wehave that the endogenous analgesia systems are much more neurochemically complex than merely opiatergic.

Methysergide induced some behavioral effects that deserve mention because of their possible confound in this study. In 4 of the 12 rats (including pilot animals) that were given the drug, it appeared to induce a "stretching" movement along the length of the rat's body. In the 30min period following methysergide administration, short bouts of stretching might be observed five or six times. This suggests a motor effect associated with the antagonist. However, Abbott, Franklin, Ludwick, and Melzack (1981) have reported that large differences in activity levels produced pharmacologically do not interfere with the baseline pain scores in the formalin test.

In order to further examine the question of motor biases associated with injection of these chemical antagonists, a test for drug-induced changes in motor ability was conducted. The rats were observed for their ability to cling to a vertically held metal grid, and no change was noted following the administration of either antagu nist. However, electrical stimulation of the PAG markedly hindered the animals' ability to cling. Under conditions without brain stimulation, most rats were frequently able to cling to the grid, even when it was held upside down; during brain stimulation, the rats lost the ability to cling when the grid advanced past the vertical position. This effect was not altered following the administration of either antagonist. To minimize the effects of motor bias due to focal brain stimulation, observations for analgesia were made after brain stimulation had been terminated.

The data indicate that the type of nociceptive challenge influences results regarding the chemical attentuation of SPA. While both tonic and phasic pain are inhibited by serotonergic action, phasic pain is also influenced by the naloxone-reversible endogenous opiate system. The results of this experiment suggest that both serotonin and the endorphins are involved in endogenous pain inhibitory systems activated by electrical stimulation of the PAG.

\section{REFERENCES}

Aвbott, F. V., Franklin, B. J., Ludwick, R. J., \& Melzack, R. (1981). Apparent lack of tolerance in the formalin test suggests different mechanisms for different types of pain. Pharmacology, Biochemistry and Behavior, 15, 637-640.

AKIL, H., \& MAYER, D. J. (1972). Antagonism of stimulation-produced analgesia by p-CPA, a serotonin synthesis inhibitor. Brain Research, 44, 692-697

Akil, H., Mayer, D. J., \& Liebeskind, J. (1976). Antagonism of stimulation-produced analgesia by naloxone, a narcotic antagonist. Science, 191, 961-962.

BenNetT, G. J., \& MAYER, D. J. (1979). Inhibition of spinal cord interneurons by the narcotic microinjection and focal brain stimulation in the periaqueductal central gray matter. Brain Research, 172, 243-257.

Cannon, J. T., Prieto, G. J., Lee, A., \& Liebeskind, J. C. (1982). Evidence for opioid and non-opioid forms of stimulation-produced analgesia in the rat. Brain Research, 243, 315-321.

D'Amour, F., \& SMITH, C. (1941). A method for determining loss of pain sensation. Joumal of Pharmacology and Experimental Therapeutics, 72, 74-79.

Dennis, S. G., Choiniere, M., \& Melzack, R. (1980). Stimulationproduced analgesia in rats: Assessment by two pain tests and correlation with self-stimulation. Experimental Neurology, 68, 295-309.

DenNis, S. G., \& Melzack, R. (1980). Comparison of phasic and tonic pain in animals. In J. J. Bonica, J. C. Liebeskind, \& D. Albe-Fessard (Eds.), Advances in pain research and therapy (Vol. 3). New York: Raven Press.

Dubuisson, D., \& DenNis, S. G. (1977). The formalin test: A quantitative study of the analgesic effects of morphine, meperidine, and brainstem stimulation in rats and cats. Pain, 4, 161-174.

HuGHES, J. (1975). Isolation of an endogenous compound from the brain with pharmacological properties similar to morphine. Brain Research, 88, 295-308.

MAYER, D., \& HAYES, R. (1975). Stimulation-produced analgesia: Development of tolerance and cross-tolerance to morphine. Science, 188, 941-943.

Pellegrino, L., \& Cushman, A. (1967). A stereotaxic atlas of the rat brain. New York: Appleton-Century-Crofts.

Prieto, G. J., Cannon, J. T., \& Liebeskind, J. C. (1983). N. raphe magnus lesions disrupt stimulation-produced analgesia from ventral but not dorsal midbrain areas in the rat. Brain Research, 261, 53-57.

REYNOLDS, D. V. (1969). Surgery in the rat during electrical analgesia induced by focal brain stimulation. Science, 164, 444-445.

Swajkoski, A. R., Mayer, D. J., \& Johnson, J. H. (1981). Blockade by naltrexone of analgesia produced by stimulation of the dorsal raphe nucleus. Pharmacology, Biochemistry and Behavior, 15, 419-423.

Thorn-Gray, B. E., Johnson, M. H., \& Ashbrook, R. M. (1982). 
Cross-tolerance between two brainstem sites supporting stimulationproduced analgesia. Behavioral and Neural Biology, 36, 69-76.

Williams, D. A., \& ThORN, B. E. (1984). Opiate withdrawal behavior after focal brain stimulation. Pharmacology, Biochemistry and Behavior, 21, 699-703.

YAKSH, T. L. (1979). Direct evidence that spinal serotonin and noradrenaline terminals mediate the spinal antinociceptive effects of morphine in the periaqueductal gray. Brain Research, 106, 180-185.
YaKsh, T. L., Yeung, J. C., \& Rudy, T. A. (1976). An inability to antagonize with naloxone the elevated nociceptive thresholds resulting from electrical stimulation of the mesencephalic central gray. Life Science, 18, 1193-1198.

(Manuscript received July 24, 1984; revision accepted for publication January 12,1985 .) 